\title{
Population-specific Mini Nutritional Assessment effectively predicts the nutritional state and follow-up mortality of institutionalized elderly Taiwanese regardless of cognitive status
}

\author{
Alan C. Tsai ${ }^{1,2} *$ and Pei-Yu Ku ${ }^{2}$ \\ ${ }^{1}$ Human Nutrition Program, Department of Environmental Health Sciences, School of Public Health, University of Michigan, \\ Ann Arbor, MI 48109, USA \\ ${ }^{2}$ Graduate Institute of Long-Term Care, Asia University, 500 Liufeng Road, Wufeng, Taichung 41354, Taiwan
}

(Received 20 April 2007 - Revised 23 August 2007 - Accepted 6 September 2007 - First published online 6 December 2007)

\begin{abstract}
The study was conducted to determine the effectiveness of a modified Mini Nutritional Assessment (MNA) for assessing the nutritional status and predicting follow-up mortality of institutionalized elderly Taiwanese. The study was conducted in a large long-term care centre in central Taiwan. Trained interviewers assisted by the caregivers elicited sociodemographic data, healthcare and disease history, and answers to the MNA screen from each subject. One researcher performed all subjects' anthropometric measurements. Plasma albumin and cholesterol concentrations were determined. Results showed that the MNA without BMI, modified according to population-specific mid-arm circumference and calf circumference cut-points, effectively predicted the nutritional risk status of the elderly regardless of cognitive status. Substituting caregiver's assessments for selfviewed nutrition and health status (questions $\mathrm{O}$ and $\mathrm{P}$ of MNA) improved the predicting power of the tool in cognition-normal subjects. Results showed that $21.9 \%$ of the elderly were malnourished, $59.2 \%$ were at risk of malnutrition and $18.9 \%$ were normal according to self-assessment whereas $14.2 \%$ were malnourished, $59.2 \%$ were at risk of malnutrition, and $26.6 \%$ were normal according to caregiver's evaluation. The tool was also effective in predicting 12- and 6-month follow-up mortality in cognition-normal and cognition-impaired elderly, respectively. Results indicate that a population-specific MNA can effectively predict the nutritional status and 6-month follow-up mortality of elderly Taiwanese regardless of cognitive condition. Easier and wider application of the tool will enable early detection of emerging nutritional problems and timely intervention to prevent the development of severe malnutrition in the elderly.
\end{abstract}

Nutritional assessment: Nutritional status: Elderly: Mortality

Malnutrition is common among frail elderly whether living at home or in care institutions. Poor nutrition can weaken one's immune competency and increase disease risk and mortality. Nutritional intervention can be effective in alleviating malnutrition, minimizing disease risk, reducing hospital stay and improving the quality of life of the elderly. To identify the malnourished elderly or individuals at risk of malnutrition, an appropriate nutritional assessment tool is often required. The Mini Nutritional Assessment (MNA) is one of the most widely used tools for such a purpose. The MNA is a simple, easy to use and non-invasive tool. The tool was developed using data generated from geriatric patients in the United States and Europe and validated with clinical data of Caucasian populations ${ }^{(1-3)}$. The tool has been shown to be useful for grading nutritional status and for predicting long-term mortality for geriatric patients entering hospitals and for elderly living at home or in care institutions ${ }^{(4-10)}$. The MNA has been used in hundreds of studies and translated into more than twenty languages ${ }^{(3)}$. However, in its original form, the tool has significant limitations in its application to non-Western, non-Caucasian groups of elderly. It has been pointed out that for it to be a truly useful screening tool for the non-Caucasian populations, the tool needs to be modified using as much countryor region-specific data and criteria as possible on the form ${ }^{(11)}$. In a previous study, the criteria for anthropometric parameters including BMI, mid-arm circumference (MAC), and calf circumference (CC), have been modified according to population-specific values based on data derived from a population-representative sample in Taiwan ${ }^{(12)}$. Modifications were also made to the diet-related questions in the MNA screen to reflect the dietary habit of the Taiwanese. The current study was conducted to evaluate the functionality of the modified MNA in elderly of varying cognitive status in a large long-term care institution in Taiwan. The study also compared caregiver's answers on two self-view questions ( $\mathrm{O}$ and $\mathrm{P}$ for nutritional and health conditions, respectively) to patients' self-evaluations. Caregiver's assessment, if proven to be acceptable for replacing self-assessment, will greatly enhance the usefulness of the tool, resulting in improvement in patient care and enhancement of job efficiency of the primary care professionals. 


\section{Subjects and methods}

The study was conducted in a large long-term care institution in Central Taiwan. Of the 351 residents, 308 (125 men and 183 women) qualified and participated in the study. Residents, 65 years or older with a minimum of 3-month of residency at the centre, were allowed to participate. Residents who had acute infections or illness, or were hospitalized during the study period were excluded from the study. A trained interviewer administered an on-site, face-to-face, interview to elicit participants' sociodemographic status, health condition, long-term care and hospitalization-related information, and the responses to the questions in the MNA screen ${ }^{(12)}$. For residents who had cognitive disorders, mental disabilities or communication difficulties, the caregivers were allowed to assist in answering the questions. For comparison purposes, residents who were cognitively competent, the answers to questions $\mathrm{O}$ and $\mathrm{P}$ for 'self-viewed' health and nutrition conditions were evaluated in two ways, by patient's view and by the caregiver's evaluations, respectively. All participants' MAC, CC, and waist circumference (WC), were measured. Additional information on participants' disease history and hospital stay was obtained from records maintained at the institution. Of the 308 participants 169 were cognitively competent to provide answers to questions $\mathrm{O}$ and $\mathrm{P}$ and were referred to as the 'cognition-competent' or 'cognition-normal' subjects. The remaining 139 elderly could not provide meaningful answers to these two questions and were considered 'cognition-impaired'.

Measurements of MAC, CC and WC were carried out with a flexible but non-stretchable measuring tape and were recorded to $0.1 \mathrm{~cm}$. All measurements followed standard procedures but with subjects in supine positions ${ }^{(13)}$. In order to avoid interrater variations (such as variation in measuring position or tightness of the tape) in anthropometric measurements, one technician performed all measurements. A blood specimen of approximately $10 \mathrm{ml}$ was taken from each participant under an over-night fasting condition on a separate day but within one week of the interview for the determination of plasma albumin and cholesterol concentrations. All biochemical assays were performed with standardized procedures by a governmentapproved clinical laboratory (Sing Chung San Clinical Laboratory, Chang-Hua, Taiwan).

In this study, the nutritional risk status of each participant was evaluated with a modified version of the MNA, using population-specific MAC and $\mathrm{CC}$ cut-points instead of values specified in the original MNA ${ }^{(12)}$. Question F (BMI) was not included because weight and height were not measured. The weighted score of MAC was adjusted from one to two points and $\mathrm{CC}$ from one to three points to cover the weighted score attributed by BMI in the original scale in consideration of the meaning of these indicators and in order to maintain the same total MNA score (thirty points). The detail of the assigned scores is shown in Table 1. Graded increments of one point per $\mathrm{cm}$ above the minimum cut values were applied to the modified MAC and CC scales $^{(14)}$. The minimum cut-values for the modified MAC and $\mathrm{CC}$ scales are the fifth percentile values of the population-representative MAC and $\mathrm{CC}$ distributions of the elderly Taiwanese ${ }^{(12)}$. For cognition-normal subjects, the total MNA score of each participant was calculated in two ways, 'selfassessed' and 'caregiver-assessed'. All other questions were the same for both methods. For cognition-impaired subjects, all questions were caregiver-assessed. The Institutional Review Board of Asia University approved the study protocol. Subject's confidentiality was maintained throughout the study.

Results were statistically analysed with Statistical Package for the Social Sciences Base 10.0 Application Guide, 1999 (SPSS Inc. Chicago, IL, USA). The first and second 6-month follow-up survival or mortality were statistically analysed. Pearson's correlation analyses were performed to determine the correlation of the total MNA scores with each of the anthropometric values, serum albumin, cholesterol, hospital length-ofstay and mode of feeding. To avoid the condition that the independent variable being tested is a component of the dependent variable, the MNA was devoid of the variable when the respective correlation was analysed. Cox regression was performed to examine the association of nutritional status, cognitive function and assessment method with the time to death from the time of baseline nutritional assessment. A $5 \%$ probability

Table 1. Anthropometric scales as specified in the original Mini Nutritional Assessment (MNA) or the modified scales for elderly Taiwanese men and women

\begin{tabular}{|c|c|c|c|c|c|}
\hline \multirow[b]{3}{*}{ Question in MNA scale } & & & \multicolumn{3}{|c|}{ Modified MNA } \\
\hline & \multicolumn{2}{|c|}{ Original MNA } & \multicolumn{2}{|c|}{ Cut-point* } & \multirow[b]{2}{*}{ Score } \\
\hline & Cut-point & Score & Men & Women & \\
\hline \multirow[t]{4}{*}{ F. BMI $\left(\mathrm{kg} / \mathrm{m}^{2}\right)$} & $<19$ & 0 & (not used) & & \\
\hline & $19-20$ & 1 & & & \\
\hline & $21-23$ & 2 & & & \\
\hline & $\geq 23$ & 3 & & & \\
\hline \multirow[t]{3}{*}{ O. Mid-arm circumference $(\mathrm{cm})$} & $<21$ & 0 & $<22.5$ & 21 & 0 \\
\hline & $21-22$ & 0.5 & $22.5-23.5$ & $21-22$ & 1 \\
\hline & $\geq 22$ & 1 & $\geq 23.5$ & 22 & 2 \\
\hline \multirow[t]{4}{*}{ P. Calf circumference $(\mathrm{cm})$} & $<31$ & 0 & $<28$ & 25 & 0 \\
\hline & $\geq 31$ & 1 & $28-28 \cdot 9$ & $25-25 \cdot 9$ & 1 \\
\hline & & & $29-29 \cdot 9$ & $26-26 \cdot 9$ & 2 \\
\hline & & & $\geq 30$ & 27 & 3 \\
\hline
\end{tabular}

\footnotetext{
* Revised according to population-specific mid-arm circumference and calf-circumference cut-points ${ }^{(12)}$. Scores adjusted to compensate for the deletion of BMI scores.
} 
$(P=0.5)$ was designated as the level of statistical significance but higher levels of significance were also indicated.

\section{Results}

The characteristics of subjects are shown in Table 2. Those who were cognition-impaired were about 2.5 years older and had more types of disabilities, lower serum albumin and total cholesterol levels and smaller $\mathrm{CC}$ and WC compared to the cognition-normal subjects (all $P<0 \cdot 05$ ). Women who were cognition-impaired also had smaller MAC, but this difference was not significant in men. Greater proportions of the cognition-impaired elderly were tube-fed or needing assistance in order to eat or were bed-ridden compared to the cognition-normal subjects (both $P<0.001$ ).

The distributions of self-assessed and caregiver-assessed nutrition and health conditions (questions $\mathrm{O}$ and $\mathrm{P}$ ) are shown in Table 3. Compared to assessments made by their caregivers, more elderly self-viewed their nutritional status as poor or were unsure of their nutritional status $(P<0.01)$. More elderly also assessed their health status as not as good as their peers or were unsure about their health status compared to assessments made by the caregivers $(P<0 \cdot 01)$

Results of nutritional status classified according to the two assessment methods and the first and the second 6-month follow-up mortality are shown in Table 4. Among the 169 elderly who were cognition-normal, $21.9 \%$ were rated malnourished, $59.2 \%$ at risk of malnutrition and $18.9 \%$ normal according to self-assessment. The respective values were $14.2 \%, 59.2 \%$ and $26.6 \%$ according to caregiver's evaluations. Of the 169 elderly, six died during the first 6-month follow-up period and thirty-three during the second 6-month period. The differences in the distribution of nutritional status among those who died between the two assessment methods were not significant. Of the 139 cognition-impaired elderly, forty-seven were rated malnourished, seventy-five at risk of malnutrition and seventeen normal. This distribution pattern is significantly different from that of the cognition-competent elderly. Seventeen of the 139 died during the first 6-month follow-up period and ten during the second 6-month period.

Table 5 shows the correlations of the total MNA scores with anthropometric, biochemical and health indicators, respectively. In order to avoid the condition that a variable being examined for the correlations is a component of the dependent variable, the total MNA score, care was taken to remove the contributing score of MAC and $\mathrm{CC}$ from the total MNA scores when testing the correlation of total MNA scores with MAC and CC, respectively. Pearson's correlation analysis showed that in cognitive-normal subjects, caregiver-assessed MNA status correlated better with all variables examined except age and serum cholesterol compared to correlations with self-assessed MNA scores. In cognition-impaired elderly, the correlation of the MNA

Table 2. Characteristics of cognition-normal ( $n$ 169) and cognition-impaired ( $n$ 139) elderly subjects

\begin{tabular}{|c|c|c|c|c|c|c|c|c|c|}
\hline \multirow[b]{2}{*}{ Item } & \multicolumn{4}{|c|}{ Cognition-normal } & \multicolumn{4}{|c|}{ Cognition-impaired } & \multirow[b]{2}{*}{$P^{*}$} \\
\hline & $n$ & $\%$ & Mean & SD & $n$ & $\%$ & Mean & SD & \\
\hline \multicolumn{10}{|l|}{ Gender } \\
\hline Male & 76 & $45 \cdot 0$ & & & 49 & $35 \cdot 3$ & & & \\
\hline Female & 93 & $55 \cdot 0$ & & & 90 & $64 \cdot 7$ & & & NS \\
\hline Age (years) & & & 79.6 & $7 \cdot 6$ & & & $82 \cdot 2$ & $7 \cdot 9$ & $<0.01$ \\
\hline \multicolumn{10}{|l|}{ Years of education } \\
\hline$\leq 6$ & 156 & $92 \cdot 3$ & & & 125 & 89.9 & & & \\
\hline $7-9$ & 10 & 5.9 & & & 6 & $4 \cdot 3$ & & & \\
\hline $10-12$ & 2 & $1 \cdot 2$ & & & 5 & $3 \cdot 6$ & & & \\
\hline$>12$ & 1 & 0.6 & & & 3 & $2 \cdot 2$ & & & NS \\
\hline \multicolumn{10}{|l|}{ Mode of feeding } \\
\hline Self-feeding & 149 & $88 \cdot 2$ & & & 49 & $35 \cdot 3$ & & & \\
\hline Need help & 17 & $10 \cdot 1$ & & & 31 & $22 \cdot 3$ & & & \\
\hline Tube-fed & 3 & 1.8 & & & 59 & $42 \cdot 4$ & & & $<0.001$ \\
\hline \multicolumn{10}{|l|}{ Mobility } \\
\hline Bed-ridden & 23 & $13 \cdot 6$ & & & 81 & $58 \cdot 3$ & & & \\
\hline Wheel-chair-bound & 108 & 63.9 & & & 50 & $36 \cdot 0$ & & & \\
\hline Needing a walker & 11 & 6.5 & & & 4 & $2 \cdot 9$ & & & \\
\hline Able to walk freely & 27 & $16 \cdot 0$ & & & 4 & 2.9 & & & $<0.001$ \\
\hline Number of disability & & & $1 \cdot 8$ & $1 \cdot 0$ & & & $3 \cdot 1$ & $1 \cdot 0$ & $<0.001$ \\
\hline \multicolumn{10}{|l|}{ Mid-arm circumference (cm) } \\
\hline Men & & & $24 \cdot 8$ & $3 \cdot 3$ & & & $24 \cdot 1$ & $2 \cdot 8$ & NS \\
\hline Women & & & $23 \cdot 6$ & $3 \cdot 3$ & & & $22 \cdot 2$ & $4 \cdot 1$ & $<0.01$ \\
\hline \multicolumn{10}{|l|}{ Calf circumference $(\mathrm{cm})$} \\
\hline Men & & & $29 \cdot 3$ & $4 \cdot 1$ & & & $27 \cdot 7$ & $3 \cdot 2$ & $<0.05$ \\
\hline Women & & & $27 \cdot 8$ & 3.9 & & & $24 \cdot 2$ & 3.8 & $<0.001$ \\
\hline \multicolumn{10}{|l|}{ Waist circumference (cm) } \\
\hline Men & & & $82 \cdot 2$ & $10 \cdot 6$ & & & $77 \cdot 8$ & $10 \cdot 4$ & $<0.05$ \\
\hline Women & & & 85.4 & $10 \cdot 9$ & & & $77 \cdot 5$ & $10 \cdot 4$ & $<0.001$ \\
\hline Serum albumin (g/dl) & & & $3 \cdot 8$ & 0.3 & & & 3.6 & 0.3 & $<0.001$ \\
\hline Total cholesterol $(\mathrm{mmol} / \mathrm{l})$ & & & $4 \cdot 7$ & $1 \cdot 0$ & & & $4 \cdot 3$ & 1.0 & $<0.001$ \\
\hline
\end{tabular}

${ }^{*} P$ values are on basis of $\chi^{2}$ analysis for gender, education, mode of feeding and mobility. All others are on basis of $t$ test. †Including immobility, incontinence, delirium and swallowing difficulty. 
Table 3. 'Self assessed' health and nutritional status (Questions $\mathrm{O}$ and $\mathrm{P}$ ), according to subjects' or caregivers' assessments

\begin{tabular}{|c|c|c|c|c|c|c|c|}
\hline \multirow[b]{3}{*}{ Question } & \multirow[b]{3}{*}{ Score } & \multicolumn{4}{|c|}{ Cognition-normal } & \multirow{2}{*}{\multicolumn{2}{|c|}{$\frac{\text { Cognition-impaired }}{\text { Caregiver-assessed }}$}} \\
\hline & & \multicolumn{2}{|c|}{ Self-assessed } & \multicolumn{2}{|c|}{ Caregiver-assessed } & & \\
\hline & & $n$ & $\%$ & $n$ & $\%$ & $n$ & $\%$ \\
\hline \multicolumn{8}{|l|}{ O. Nutritional status } \\
\hline Poor & 0 & 65 & 38.5 & 18 & $10 \cdot 7$ & 24 & $17 \cdot 3$ \\
\hline Not sure & 1 & 59 & 34.9 & 39 & $23 \cdot 1$ & 30 & $21 \cdot 6$ \\
\hline No nutritional problem & 2 & 45 & $26 \cdot 6$ & $112^{*}$ & $66 \cdot 3$ & $85 \dagger$ & $61 \cdot 2$ \\
\hline \multicolumn{8}{|l|}{ P. Health status } \\
\hline Not as good & 0 & 53 & 31.4 & 15 & 8.9 & 40 & $28 \cdot 8$ \\
\hline Does not know & 0.5 & 18 & $10 \cdot 7$ & 5 & $3 \cdot 0$ & 10 & $7 \cdot 2$ \\
\hline As good & 1 & 50 & $29 \cdot 6$ & 89 & 52.7 & 67 & $48 \cdot 2$ \\
\hline Better & 2 & 48 & 28.4 & $60^{*}$ & 35.5 & $22 \dagger$ & $15 \cdot 8$ \\
\hline
\end{tabular}

* The distribution of nutritional status is significantly different from that of the self-assessed $(P<0.01)$.

$\dagger$ The distribution of nutritional status is significantly different from that of the cognition-normal elderly $(P<0.01)$.

score with age, MAC and $\mathrm{CC}$ were stronger compared to cognition-normal elderly whereas the correlations of the MNA score with WC, albumin, total cholesterol, hospital length-of-stay and mode of feeding were weaker.

Fig. 1 shows the survival curves and the associations of nutritional status, cognitive function and assessment method of patients with the time to death from the time of nutritional assessment. Log-rank (Mantel-Cox) tests showed significant differences in 12-month survival between normal and at risk $(P<0.01)$ and malnourished $(P<0.05)$ groups in cognition-normal elderly assessed by the caregivers but not by self-assessment. Significant differences also existed in 6-month but not 12-month survival between the normal and at risk $(P<0.01)$ and malnourished $(P<0.01)$ groups in cognition-impaired elderly assessed by their caregivers.

\section{Discussion}

The MNA has been intended to provide the primary care health professionals with an efficient tool for identifying the elderly individuals malnourished or at risk of malnutrition. It was designed to assess nutritional status of frail elderly individuals having some functional impairment such as immobility, hearing, speech and cognitive disorders, and living alone or in nursing homes, or being hospitalized ${ }^{(6)}$. The tool assesses one's nutritional status in four dimensions: anthropometric measurements including weight changes and body muscle mass; global assessment including lifestyle, medication and mobility status; dietary assessment to indicate intake of fluid and important nutrients and feeding status; and subjective self-perception of health and nutrition status. Since elderly who have cognitive disorders or dementia will have impaired ability to answer the questions in the MNA, it has been suggested that in those individuals the health professional (the interviewer) must score the MNA with the caregiver or based on his/her own judgement of the conditions ${ }^{(6)}$. According to our experience, to the cognitively less competent elderly the two self-assessed questions are among the questions with which they often encounter the greatest difficulties. The frail or cognitively less competent elderly, especially those who have had little formal education, have difficulty in both comprehending and answering these two questions. Thus, a caregiver-assessed MNA, if effective, will greatly enhance the usefulness of the tool.

Table 4. First and second follow-up 6-month total mortality of cognition-normal and cognition-impaired elderly based on nutritional risk status classified according to the original or the modified MNA

\begin{tabular}{|c|c|c|c|}
\hline \multirow[b]{2}{*}{ Item } & \multicolumn{3}{|c|}{ Nutritional risk status } \\
\hline & Malnourished & At risk of malnutrition & Normal \\
\hline \multicolumn{4}{|c|}{ Cognition normal, self-assessed ( $n$ 169) } \\
\hline $\begin{array}{l}n \text { in group } / n \text { total } \\
n \text { death } / n \text { in group }\end{array}$ & $37 / 169(21.9 \%)$ & $100 / 169(59 \cdot 2 \%)$ & $32 / 169(18.9 \%)$ \\
\hline First 6 months & $2 / 37(5.4 \%)$ & $3 / 100(3.0 \%)$ & $1 / 32(3 \cdot 1 \%)$ \\
\hline Month 7-12 & $2 / 37(5.4 \%)$ & $9 / 100(9.0 \%)$ & $0 / 32(0 \%)$ \\
\hline \multicolumn{4}{|c|}{ Cognition normal, caregiver-assessed ( $n$ 169) } \\
\hline $\begin{array}{l}n \text { in group } / n \text { total } \\
n \text { death } / n \text { in group }\end{array}$ & $24 / 169(14.2 \%)$ & $100 / 169(59 \cdot 2 \%)$ & $45 / 169(26 \cdot 6 \%)$ \\
\hline First 6 months & $1 / 24(4 \cdot 2 \%)$ & $5 / 100(5.0 \%)$ & $0 / 45(0 \%)$ \\
\hline Month 7-12 & $2 / 24(8.3 \%)$ & $9 / 100(9.0 \%)$ & $0 / 45(0 \%)$ \\
\hline \multicolumn{4}{|c|}{ Cognition-impaired, caregiver-assessed (n 139) } \\
\hline $\begin{array}{l}n \text { in group } / n \text { total } \\
n \text { death } / n \text { in group }\end{array}$ & $47 / 139(33.8 \%)$ & $75 / 139(54.0 \%)$ & $17 / 139(12 \cdot 2 \%)^{\star}$ \\
\hline First 6 months & $7 / 47(14.9 \%)$ & $10 / 75(13.3 \%)$ & $0 / 17(0 \%)$ \\
\hline Month 7-12 & $3 / 47(6.4 \%)$ & $4 / 75(5.3 \%)$ & $3 / 17(17.6 \%)$ \\
\hline
\end{tabular}

* The distribution of nutrition status was significantly different $(P<0.05)$ from that of the cognition-normal elderly on basis of $\chi^{2}$ test. 
Table 5. Correlations of self-assessed and caregiver-assessed total Mini Nutritional Assessment (MNA) scores (revised scale) with anthropometric, albumin and health status in cognition-normal and cognition-impaired elderly

\begin{tabular}{|c|c|c|c|c|c|c|}
\hline & \multicolumn{4}{|c|}{ Cognition-normal ( $n$ 169) } & \multirow{2}{*}{\multicolumn{2}{|c|}{$\frac{\text { Cognition-impaired ( } n \text { 139) }}{\text { Caregiver-assessed }}$}} \\
\hline & \multicolumn{2}{|c|}{ Self-assessed } & \multicolumn{2}{|c|}{ Caregiver-assessed } & & \\
\hline & $r$ & $P$ & $r$ & $P$ & $r$ & $P$ \\
\hline Age & -0.190 & 0.013 & -0.167 & 0.030 & -0.228 & 0.007 \\
\hline Waist circumference & 0.408 & $<0.0001$ & 0.461 & $<0.0001$ & 0.375 & $<0.0001$ \\
\hline $\mathrm{MAC}^{\star}$ & 0.244 & 0.001 & 0.355 & $<0.0001$ & 0.485 & $<0.0001$ \\
\hline $\mathrm{CC}^{*}$ & 0.255 & 0.001 & 0.364 & $<0.0001$ & 0.395 & $<0.0001$ \\
\hline Albumin & 0.299 & $<0.0001$ & 0.333 & $<0.0001$ & 0.254 & 0.005 \\
\hline Total cholesterol & 0.245 & 0.002 & 0.231 & 0.003 & 0.032 & 0.729 \\
\hline Hospital length-of-stay & -0.216 & 0.006 & -0.265 & 0.001 & -0.257 & 0.003 \\
\hline Mode of feeding $\dagger$ & 0.300 & 0.169 & 0.324 & $<0.0001$ & 0.191 & 0.025 \\
\hline
\end{tabular}

MAC, mid-arm circumference; CC, calf circumference.

* In order to avoid the condition where the independent variable is a component of the dependent variable, the scores of MAC and CC were removed from total MNA scores when testing for its correlation with MAC and CC, respectively.

†Categorically whether self-fed, need assistance or tube-fed.

Results of the present study indicate that under the condition that everything else remains the same, the caregivers can provide a more effective 'self-evaluation' of patient's nutritional and health conditions (questions $\mathrm{O}$ and $\mathrm{P}$ ) compared to patient's self-evaluations. This is evidenced by improved correlation of the total MNA score with each of the health indicators including the 'gold standard', serum albumin. Caregiver's assessment is also more effective in predicting follow-up mortality compared to patient-assessed MNA. The caregiver-assessed MNA predicted all seventeen deaths (six during the first 6-month and eleven during the second 6-month period) whereas the self-assessed MNA predicted sixteen of the seventeen cases during the same period. The caregiver-assessment is also effective in predicting both nutritional status and 6-month follow-up mortality in cognitionimpaired elderly. Results of Cox regression analysis of the probability of survival of the elderly subjects further supports this finding. In cognition-normal patients, significant differences in the probability of survival between the nutritionally normal and at-risk and malnourished elderly were observed only by caregiver-assessment but not by self-assessment, whereas in cognition-impaired patients, significant differences in the probability of survival between the nutritionally normal and at-risk and malnourished were also observed when assessed by the caregivers. These results indicate that using the modified MNA screen the caregiver can effectively evaluate the nutritional risk status of the elderly, regardless of their cognitive status. Caregiver's professional knowledge and experience probably contribute to the improved predicting power of the MNA. In cognition-competent elderly the predicting power seems to last beyond one whole year whereas in cognition-impaired elderly, the predicting power weakens after the first 6 months. This is probably due to generally poorer health conditions of the cognition-impaired elderly.

The MNA has been shown to have significant predicting power on mortality in a Danish population ${ }^{(15)}$ and in geriatric patients $^{(16-20)}$. Individuals scored 24 points or better had significantly lower follow-up mortality compared with those who scored less. Depending on the condition of subjects, one-year follow-up mortality varied from $<10 \%$ to as high as $50 \%$ among those who were classified malnourished (MNA score
$<17$ ). The MNA has been used as a grading scale for providing nutritional intervention to frail elderly. Individuals who score $<17$ MNA points are considered to have proteinenergy malnutrition and should receive immediate nutritional intervention otherwise their immune competency will be severely compromised whereas those scored 17 to 23.5 points are at risk of protein-energy malnutrition and should also be advised to correct potential nutritional deficiency before becoming malnourished ${ }^{(6)}$.

There are three important features in this modified MNA screen for the Taiwanese elderly population: (a) It does not require BMI values, thus measurements of height and weight are not required. Measuring weight and height may not be always possible because of lack of special equipment; and the accuracy, especially of height, is not always assured. (b) It uses population-specific MAC and $\mathrm{CC}$ cut-points and thus can presumably produce more accurate results, and (c) the tool can be caregiver-assessed and thus can be applied to both cognition-normal and cognition-impaired individuals. Those features will undoubtedly improve both the utility and the accuracy of the tool. The modified tool should be a valuable tool for monitoring nutritional status of the frail elderly whether they are home-living or institutionalized. It will enable timely nutritional intervention to correct nutritional deficiencies or to prevent developing nutritional deficiencies in those high-risk individuals.

Although the current study was conducted in a relatively large long-term care centre with mixed types of residents in Taiwan, the application of this tool to assess the nutritional risk status of elderly with specific chronic health conditions such as stroke, dementia, renal disease or cancer requires further confirmation. The effectiveness of the tool in home-living elderly also requires confirmation. It is also important to ascertain whether information obtained from close family members can be as good as the caregivers in long-term care institutions.

\section{Conclusion}

The current study has demonstrated that using a modified MNA screen the nutritional status of the institutionalized frail elderly can be effectively assessed by the caregivers. 

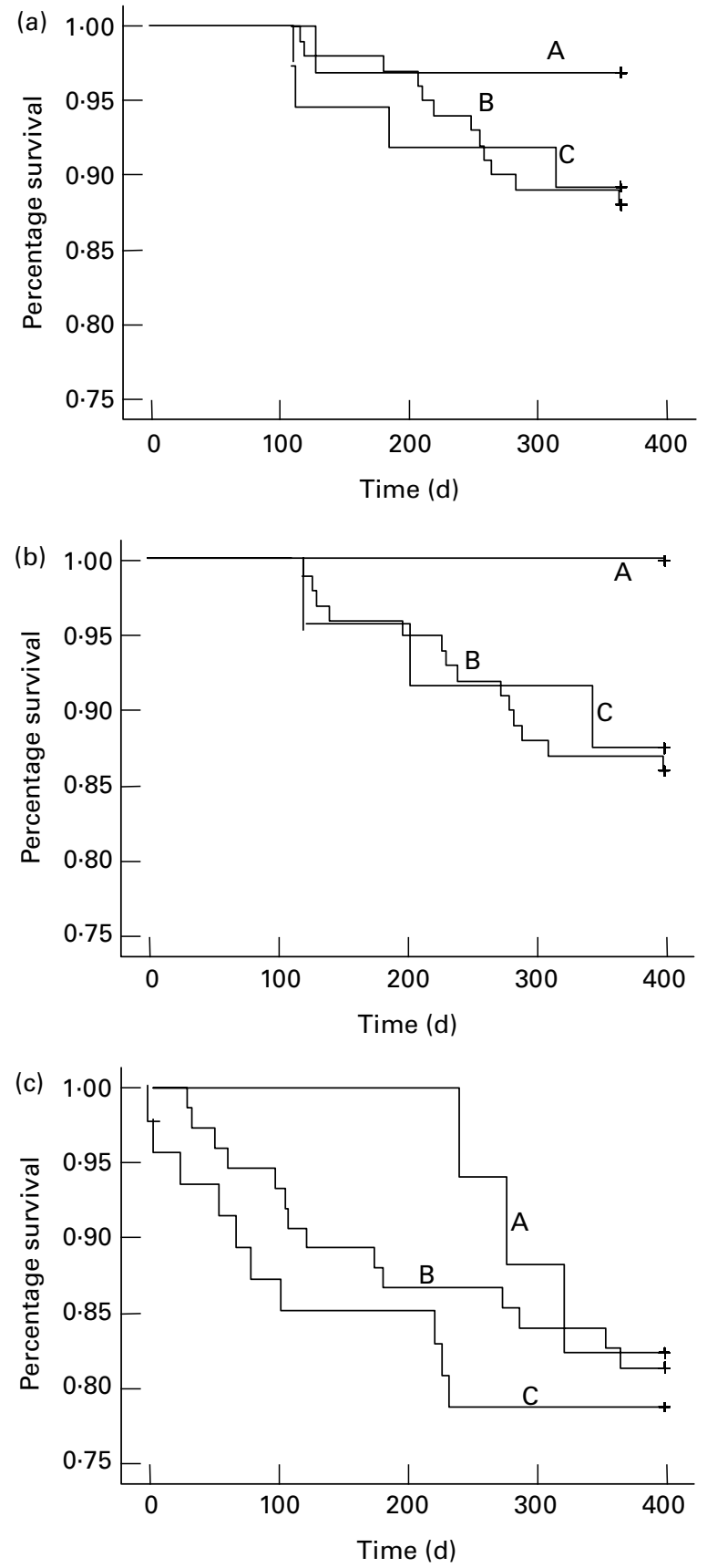

Fig. 1. Survival curves for subjects according to cognitive status $((a)$ and $(b)$ cognition-normal; (c) cognition-impaired), assessment methods ((a) selfassessed; (b) and (c) caregiver-assessed) and Mini Nutritional Assessment status at baseline. Lines A, B and C represent normal, at risk of malnutrition and malnourished status, respectively. Log-rank (Mantel-Cox) tests showed significant differences in 12-month survival between normal and at risk $(P<0.01)$ and malnourished $(P<0.05)$ groups in (b) (cognition-normal, caregiver-assessed). Significant differences were also observed in 6-month survival between normal and at risk of malnutrition $(P<0.01)$ and malnourished $(P<0.01)$ groups in (c) (cognition-impaired, caregiver-assessed).

Caregivers' assessments are more effective in predicting nutritional risk status and the 6-month follow-up mortality in institutionalized cognition-normal elderly compared to patients' self-evaluations. Results of the present study also show that using caregiver's evaluation, the MNA is effective in predicting the nutritional risk status and 6-month follow-up mortality of the cognition-impaired elderly.

\section{Acknowledgements}

Funding for this study was provided by a private donation. The authors wish to thank the caregivers and the administrators of the LTC centre for providing the assistance during the course of the study. A. C. T. conceived, directed and supervised the study and is the main writer of the manuscript. P.-Y. K. carried out the field study, performed statistical analyses and reviewed the manuscript. The authors also wish to express their appreciation to Dr H. J. Tsai for her assistance in statistical analysis. The authors also declare that there is no conflict of interest of any kind involved in this study or this publication.

\section{References}

1. Guigoz Y, Vellas BJ \& Garry PJ (1994) The Mini Nutritional Assessment (MNA): a practical assessment tool for grading the nutritional state of elderly patients. In Facts and Research in Gerontology, Nutrition Supplement, pp. 15-60 [BJ Vellas, Y Guigoz, PJ Garry and JL Albarede, editors]. New York: Serdi Publishing Co.

2. Guigoz Y, Lauque S \& Villas BJ (2002) Identifying the elderly at risk for malnutrition. The Mini Nutritional Assessment. Clin Geriatr Med 18, 737-757.

3. Vellas B, Villars H, Abellan G, et al. (2006) Overview of the MNA - its history and challenge. J Nutr Health Aging 10, 456-463.

4. Bleda MJ, Bolibar I, Pares R \& Salva A (2002) Reliability of the Mini Nutritional Assessment (MNA) in institutionalized elderly people. J Nutr Health Aging 6, 134-137.

5. Soini H, Routasalo P \& Lagstrom H (2004) Characteristics of the Mini-Nutritional Assessment in elderly home-care patients. Eur J Clin Nutr 58, 64-70.

6. Vellas B, Guigoz Y, Garry PJ, Nourhashemi F, Bennahum D, Lauque S \& Albarede JL (1999) The Mini Nutritional Assessment (MNA) and its use in grading the nutritional state of the elderly patients. Nutrition 15, 116-122.

7. Guigoz Y, Vellas B \& Garry PJ (1996) Assessing the nutritional status of the elderly: The Mini Nutritional Assessment as part of the geriatric evaluation. Nutr Rev 54, Suppl., S59-S65.

8. Delacorte RR, Moriguti JC, Motos FD, Perimer K, Marchini JS \& Ferriolli E (2004) Mini-Nutritional Assessment score and the risk for undernutrition in free-living older persons. $J$ Nutr Health Aging 8, 531-534.

9. Kuzuya M, Kanda S, Koike T, Suzuki Y, Satake S \& Iguchi A (2005) Evaluation of Mini-Nutritional Assessment for Japanese frail elderly. Nutrition 21, 498-503.

10. Izawa S, Kuzuya M, Okada K, Enoki H, Koike T, Kanda S \& Iguchi A (2006) The nutritional status of frail elderly with care needs according to the mini-nutritional assessment. Clin Nutr 25, 962-967.

11. Chumlea WC (1999) The state of the Mini Nutritional Assessment? Nutrition 15, 159-161.

12. Tsai AC, Ho CS \& Chang MC (2007) Population-specific anthropometric cut-points improve the functionality of the Mini Nutritional Assessment (MNA) in elderly Taiwanese. Asia Pac J Clin Nutr (In the Press).

13. Lee RD and Nieman DC (editors) (2003) Assessment of the hospitalized patient. In Nutritional Assessment, 3rd ed., pp. 216-250. New York: McGraw-Hill Higher Education.

14. Tsai AC, Ku PY \& Tsai JD (2007) Population specific anthropometric cutoff standards improve the functionality of the 
Mini Nutritional Assessment (MNA) in institutionalized elderly in Taiwan. $J$ Nutr Health Aging (In the Press).

15. Beck AM, Ovesen L \& Osler M (1999) The 'Mini Nutritional Assessment' (MNA) and the "Determine Your Nutritional Health" Checklist (NSI Checklist) as predictors of morbidity and mortality in an elderly Danish population. Br J Nutr 81, $31-36$.

16. Gazzotti C, Albert A, Pepinster A \& Petermans J (2000) Clinical usefulness of the mini nutritional assessment (MNA) scale in geriatric medicine. J Nutr Health Aging 4, 176-181.

17. Donini LM, Savina C, Rosano A, et al. (2003) MNA predictive value in the follow-up of geriatric patients. J Nutr Health Aging 7, 282-293.
18. Persson MD, Brismar KE, Katzarski KS, Nordenström J \& Cederholm TE (2002) Nutritional status using Mini Nutritional Assessment and Subjective Global Assessment predict mortality in geriatric patients. $J$ Am Geriatr Soc 50, 1996-2002.

19. Van Nes M-C, Herrmann FR, Gold G, Michel J-P \& Rizzou R (2001) Does the Mini Nutritional Assessment predict hospitalization outcomes in older people? Age Ageing 30, 221-226.

20. Compan B, DiCastri A, Plaze J-M \& Arnaud-Battandier F (1999) Epidemiological study of malnutrition in elderly patients in acute, sub-acute and long-term care using the MNA. J Nutr Health Ageing 3, 146-151. 